\title{
SEM and EPMA Analyses of Metallic Inclusions in Diamonds - Probing the Earth's Deep Mantle
}

\author{
Emma S. Bullock ${ }^{1}$, Evan M. Smith ${ }^{2}$ and Steven B Shirey ${ }^{3}$ \\ 1. Geophysical Laboratory, Carnegie Institution for Science, Washington, DC. \\ 2. Gemological Institute of America, New York, NY. \\ 3. Department of Terrestrial Magnetism, Carnegie Institution for Science, Washington, DC.
}

The Earth's core is made up of a solid inner portion, encircled by a liquid metallic region [Fig. 1]. Surrounding this is a thick mantle made up of silicates and minor amounts of metal (perhaps reaching 1 $\mathrm{wt} \%$ ), and the outermost layer is the crust, made up of silicates (Figure 1). The mantle is not homogeneous - there are distinct regions of differing mineralogy and composition [2]. Understanding these different regions can inform our ideas of mantle evolution; in particular the carbon cycle in the deep earth, and our knowledge of the redox state (availability of oxygen) within the mantle [3]. Accessing the mantle directly is impossible: our Earth has a radius of $\sim 6400 \mathrm{~km}$, and the deepest hole that has been dug to date is the $12 \mathrm{~km}$ deep Kola Superdeep Borehole in the Kola Peninsula, Russia, which is still within the crust. If we want to learn about the conditions in the deep Earth, then we rely on samples that were created at great depths, and then brought to the surface through geological activity. One of these materials is diamond.

Diamonds form at great temperatures and depths within the mantle. Some diamonds grow within metallic pockets in the mantle, and have the potential to trap as inclusions some of the metal-rich medium that surrounded them (Figure 2). A particular group of such diamonds - the CLIPPIR diamonds (Cullinan-like, Large, Inclusion-Poor, relatively Pure, Irregularly shaped, and Resorbed) - have been recently identified that contain metallic inclusions that are interpreted to be trapped mantle material [4]. Here we present results on the composition of these inclusions as determined by the scanning electron microscope and electron probe microanalysis, to see what they reveal about the conditions under which the diamonds formed.

The diamonds were examined optically in order to identify examples which contain inclusions. The diamonds were then polished, in order to expose the surface of each inclusion. They were then coated with a $0.6 \mathrm{~nm}$ thick layer of Ir. X-ray maps were collected using a JEOL 6500 scanning electron microscope operating at $15 \mathrm{kV}$ and $1 \mathrm{nA}$, running the Oxford Aztec software. The maps show that the inclusions are made up of variable proportions of iron carbide, iron sulfide, and Fe-Ni metal (Figure 3). Accessory minerals include iron phosphate and Ca-silicate perovskite as well as Fe- and Fe-Cr-oxides. Two inclusions of majoritic garnet trapped along with metal in a CLIPPIR diamond were also analyzed.

Mineral compositions were obtained using a JEOL 8530F electron microprobe operating at $15 \mathrm{kV}$ and 30nA. For the metal inclusions, the standards used were Fe metal, Ni metal, a synthetic iron carbide $\left(\mathrm{Fe}_{7} \mathrm{C}_{3}\right)$, troilite $(\mathrm{FeS})$ and $\mathrm{Si}$ metal. For the garnets, the standards used were almandine, spessartine, a sodium-bearing glass, augite, magnesium-bearing chromite and pyrope. The probe data reveal that the iron carbide is cohenite $\left[(\mathrm{Fe}, \mathrm{Ni})_{3} \mathrm{C}\right]$, while the iron sulfide is pyrrhotite $\left(\mathrm{Fe}_{(1-\mathrm{x})} \mathrm{S}\right)$. The garnet is $\mathrm{Cr}$-poor majoritic garnet, containing on average $20 \mathrm{wt} \% \mathrm{Al}_{2} \mathrm{O}_{3}, 13 \mathrm{wt} \% \mathrm{MgO}, 17 \mathrm{wt} \% \mathrm{FeO}$ and $44 \mathrm{wt} \% \mathrm{SiO}_{2}$ (excess Si compared to a stoichiometric garnet). 
The Fe-Ni-C-S metallic composition of the inclusions in CLIPPIR diamonds suggests that the conditions under which they formed was highly reducing - there was not much oxygen available in the regions where these diamonds formed. The inclusions are interpreted as a key physical confirmation that the deep mantle, below about $250 \mathrm{~km}$, is saturated with metallic iron, as predicted from theory and experiments. The carbon content of the metal indicates that Fe-Ni metal is capable of dissolving substantial quantities of carbon, which has implications for the storage and cycling of carbon and other light elements within the deep earth. The high-pressure silicate inclusions in CLIPPIR diamonds former Ca-silicate perovskite and majoritic garnet - constrain the formation depth of the CLIPPIR diamonds to within 360-750 km depth, while the Cr-poor composition of the garnets indicates that the source rocks were eclogitic (sourced from deep within the mantle) rather than from the peridotitic upper mantle.

References:

[1] T H Jordan, Proc. Natl. Acad. Sci 76 (1979), p.4192.

[2] D L Anderson, Tectonophysics 416 (2006), p. 7.

[3] B Scaillet and F Gaillard, Nature 480 (2011), p. 48.

[4] E M Smith et al, Science 354 (2016) p. 1403.

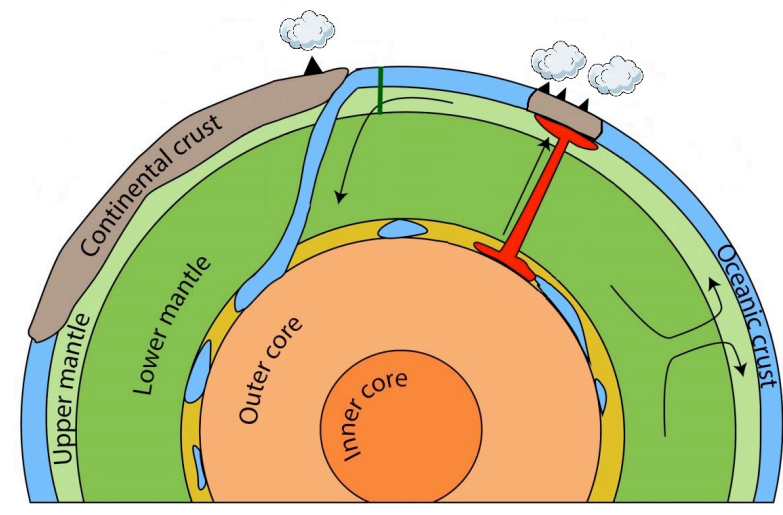

Figure 1. Cross section of the Earth, showing the core, mantle and crust. Diamonds are generated within the Earth's mantle, and brought to the surface through volcanic activity.
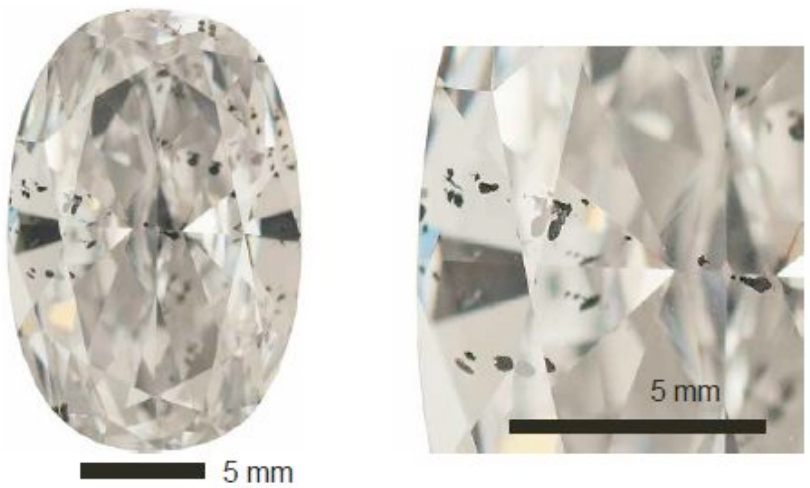

Figure 2. Cut diamond showing inclusions (left) and close-up of inclusions (right).
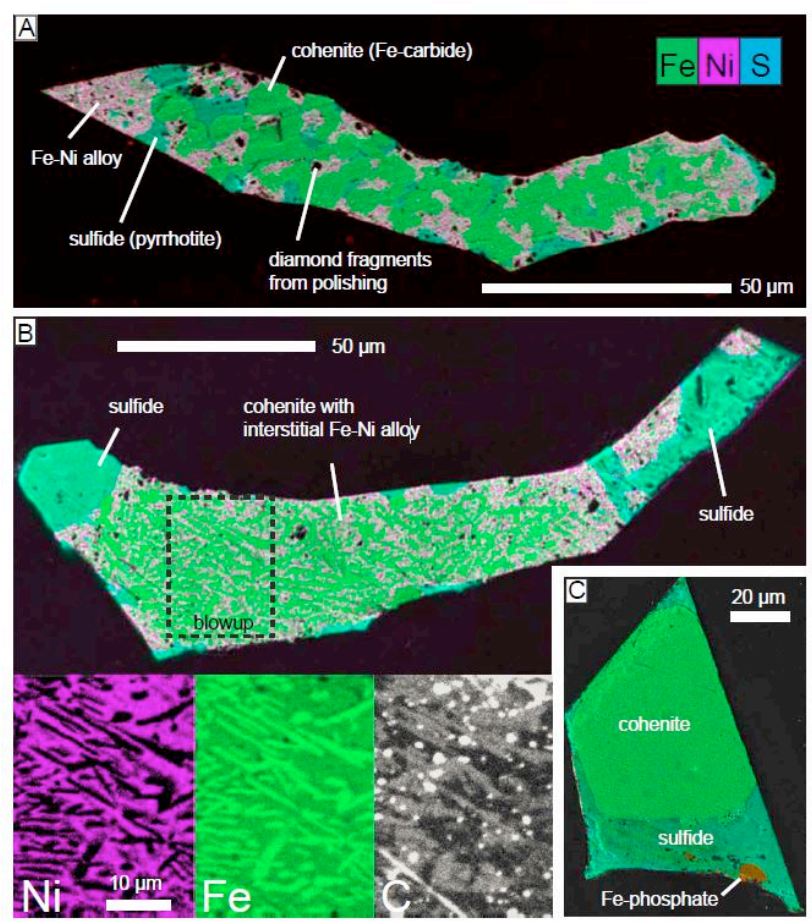

Figure 3. SEM image of inclusion, overlaid with x-ray maps of Fe (green), nickel (purple) and sulfur (blue). Cohenite $\left[(\mathrm{Fe}, \mathrm{Ni})_{3} \mathrm{C}\right]$ appears green, pyrrhotite $\left(\mathrm{Fe}_{(1-\mathrm{x})} \mathrm{S}\right)$ as light blue, and $\mathrm{Fe}$ Ni metal as pink. Detailed Ni, Fe, and $\mathrm{C}$ maps for the dashed area in (B) show that the cohenite is relatively $\mathrm{Ni}$-poor but $\mathrm{Fe}$ - and $\mathrm{C}$-rich. 